\title{
Intraarticular injection of bone marrow-derived mesenchymal stem cells enhances regeneration in knee osteoarthritis
}

\author{
Emily Claire Doyle ${ }^{1} \cdot$ Nicholas Martin Wragg ${ }^{2} \cdot$ Samantha Louise Wilson $^{2}$ (i)
}

Received: 11 July 2019 / Accepted: 16 January 2020 / Published online: 31 January 2020

(c) The Author(s) 2020

\begin{abstract}
Purpose This review aimed to evaluate the efficacy of intra-articular injections of bone marrow derived mesenchymal stem cells (BM-MSCs) for the treatment of knee osteoarthritis (KOA).

Methods This narrative review evaluates recent English language clinical data and published research articles between 2014 and 2019. Key word search strings of ((("bone marrow-derived mesenchymal stem cell” OR "bone marrow mesenchymal stromal cell" OR "bone marrow stromal cell")) AND ("osteoarthritis" OR "knee osteoarthritis")) AND ("human" OR “clinical”))) AND “intra-articular injection" were used to identify relevant articles using PMC, Cochrane Library, Web Of Science and Scopus databases.

Results Pre-clinical studies have demonstrated successful, safe and encouraging results for articular cartilage repair and regeneration. This is concluded to be due to the multilineage differential potential, immunosuppressive and self-renewal capabilities of BM-MSCs, which have shown to augment pain and improve functional outcomes. Subsequently, clinical applications of intra-articular injections of BM-MSCs are steadily increasing, with most studies demonstrating a decrease in poor cartilage index, improvements in pain, function and Quality of Life (QoL); with moderate-to-high level evidence regarding safety for therapeutic administration. However, low confidence in clinical efficacy remains due to a plethora of heterogenous methodologies utilised, resulting in challenging study comparisons. A moderate number of cells $\left(40 \times 10^{6}\right)$ were identified as most likely to achieve optimal responses in individuals with grade $\geq 2 \mathrm{KOA}$. Likewise, significant improvements were reported when using lower $\left(24 \times 10^{6}\right)$ and higher $\left(100 \times 10^{6}\right)$ cell numbers, although adverse effects including persistent pain and swelling were a consequence.

Conclusion Overall, the benefits of intra-articular injections of BM-MSCs were deemed to outweigh the adverse effects; thus, this treatment be considered as a future therapy strategy. To realise this, long-term large-scale randomised clinical trials are required to enable improved interpretations, to determine the validity of efficacy in future studies.
\end{abstract}

Level of evidence IV.

Keywords Allogenic · Autologous · Cell therapies · Clinical efficacy · Immunomodulation · Mesenchymal stem cells · Optimal dosage $\cdot$ Osteoarthritis

Samantha Louise Wilson

s.wilson2@1boro.ac.uk

1 National Centre for Sport and Exercise Medicine, School of Sport, Exercise and Health Sciences, Loughborough University, Epinal Way, Loughborough LE11 3TU, Leicestershire, UK

2 Centre for Biological Engineering, Wolfson School of Mechanical, Electrical and Manufacturing Engineering, Loughborough University, Epinal Way, Loughborough LE11 3TU, Leicestershire, UK

\begin{tabular}{|c|c|}
\hline \multicolumn{2}{|c|}{ Abbreviations } \\
\hline $\mathrm{OA}$ & Osteoarthritis \\
\hline QoL & Quality of life \\
\hline BM-MSCs & $\begin{array}{l}\text { Bone marrow derived-mesenchymal stem } \\
\text { cells }\end{array}$ \\
\hline KOA & Knee osteoarthritis \\
\hline WHO & World Health Organisation \\
\hline MMPs & Matrix metalloproteinases \\
\hline BMP2 & Bone morphogenetic protein 2 \\
\hline IGF1 & Insulin-like growth factor 1 \\
\hline IL-1 & Interleukin-1 \\
\hline ISCT & International Society for Cellular Therapy \\
\hline
\end{tabular}


WOMAC The Western Ontario and McMaster Universities Osteoarthritis Index

VAS Visual analogue scale

KOOS Knee injury and osteoarthritis outcome score

MRI Magnetic resonance imaging

PBS Phosphate-buffered saline

RCT Randomised control trial

\section{Introduction}

Osteoarthritis (OA) is one of the most ubiquitous joint disorders [1]; the prevalence of symptomatic hip and/or knee OA is $\sim 242$ million worldwide [2] with conditions ranked as the 11th highest contributors to global disability [1]. Decreased patient quality of life (QoL) and productivity poses a significant individual and societal burden, with a global prevalence of 3.8\% [3, 4]. Knee OA (KOA) demonstrates higher incidences compared to other joints, with a lifetime risk of $\sim 45 \%$, increasing to $60.5 \%$ amongst obese patients [5]. Prevalence increases with each decade of life, with annual incidences highest between the ages of 55-65 years [6-9] further exacerbated by endogenous and exogenous risk factors (Table 1).

OA results from degradation of the osteochondral unit composed of: articular cartilage; calcified cartilage; subchondral and trabecular bone, which synergistically support functional loading [10]. Throughout OA progression, degradative enzymes are overexpressed, including matrix metalloproteinases (MMPs) [11], which degrade both matrix and non-matrix proteins. Chondrocyte senescence and reduced cartilage elasticity [12] alters the tissue microenvironment impairing regeneration. Morphological changes in the subchondral bone include cartilage surface fibrillation and synovial fluid thickening [13, 14], accompanied by progressive synovitis and osteophyte formation. Progression according to imaging can be graded as localised (focal) or diffuse (affecting $\geq 75 \%$ of the region), and normal, doubtful, mild, moderate or severe (grade $0-4$ on the Kellegren Lawrence scale) [15].

Currently, no conventional or pharmacological therapies have demonstrated unequivocal efficacy in halting disease progression and injections of molecular compounds to assist healing, such as corticosteroids, may only have placebic pain reducing effects [16-18]. Surgical interventions may be beneficial when mechanical deformity is present; however, therapeutic benefit is limited to late-stage $\mathrm{OA}$ and is not considered as a long-term solution. Alternatively, cellular regenerative therapies, including mesenchymal stem cells (MSCs) [19] and cell-derived products (such as platelet-rich plasma [20]) have shown therapeutic promise. Since OA is degenerative, likely involving endogenous MSC depletion, investigation into this therapy is supported by BM-MSCs inherent characteristics (Fig. 1) and their potential for articular cartilage repair/regeneration [21].

The purpose of this study was to review published literature to assess and evaluate the clinical efficacy of intra-articular injections of bone marrow-derived MSCs (BM-MSCs) specifically for KOA. It was hypothesized that BM-MSCs would have a beneficial impact on KOA clinical outcomes. This is clinically relevant since OA poses a significant individual, societal and economic global burden.

Table 1 Endogenous and exogenous risk factors for KOA

\begin{tabular}{|c|c|}
\hline Endogenous & Exogenous \\
\hline $\begin{array}{l}\text { Age } \\
\text { Incidence rates increase linearly in the } 50-80 \text { age range }\end{array}$ & Previous joint injuries \\
\hline Sex & Body mass \\
\hline $\begin{array}{l}\text { Females have been reported to have a greater incidence rate } \\
\text { compared to males }\end{array}$ & $\begin{array}{l}\text { Overweight and obese people are significantly associated with higher KOA risk } \\
\text { The risk increases by } 35 \% \text { with every } 5 \mathrm{~kg} / \mathrm{m}^{2} \text { increase in BMI }\end{array}$ \\
\hline Heredity & Excessive joint stress and increased mechanical forces \\
\hline Genetics & Repetitive loading (kneeling and squatting) \\
\hline Joint laxity & $\begin{array}{l}\text { Occupation } \\
\text { Physical work activities (kneeling/squatting/lifting and climbing) contribute to } \\
\text { the occurrence/progression of KOA }\end{array}$ \\
\hline $\begin{array}{l}\text { Ethnic origin } \\
\text { More common in individuals of European descent }\end{array}$ & Resective joint surgery \\
\hline Post-menopausal changes & Muscle weakness \\
\hline Malalignment & Lifestyle factors (alcohol, tobacco use) \\
\hline
\end{tabular}

Adapted from: Adatia et al. [64] and supplemented by Palmer [65] 


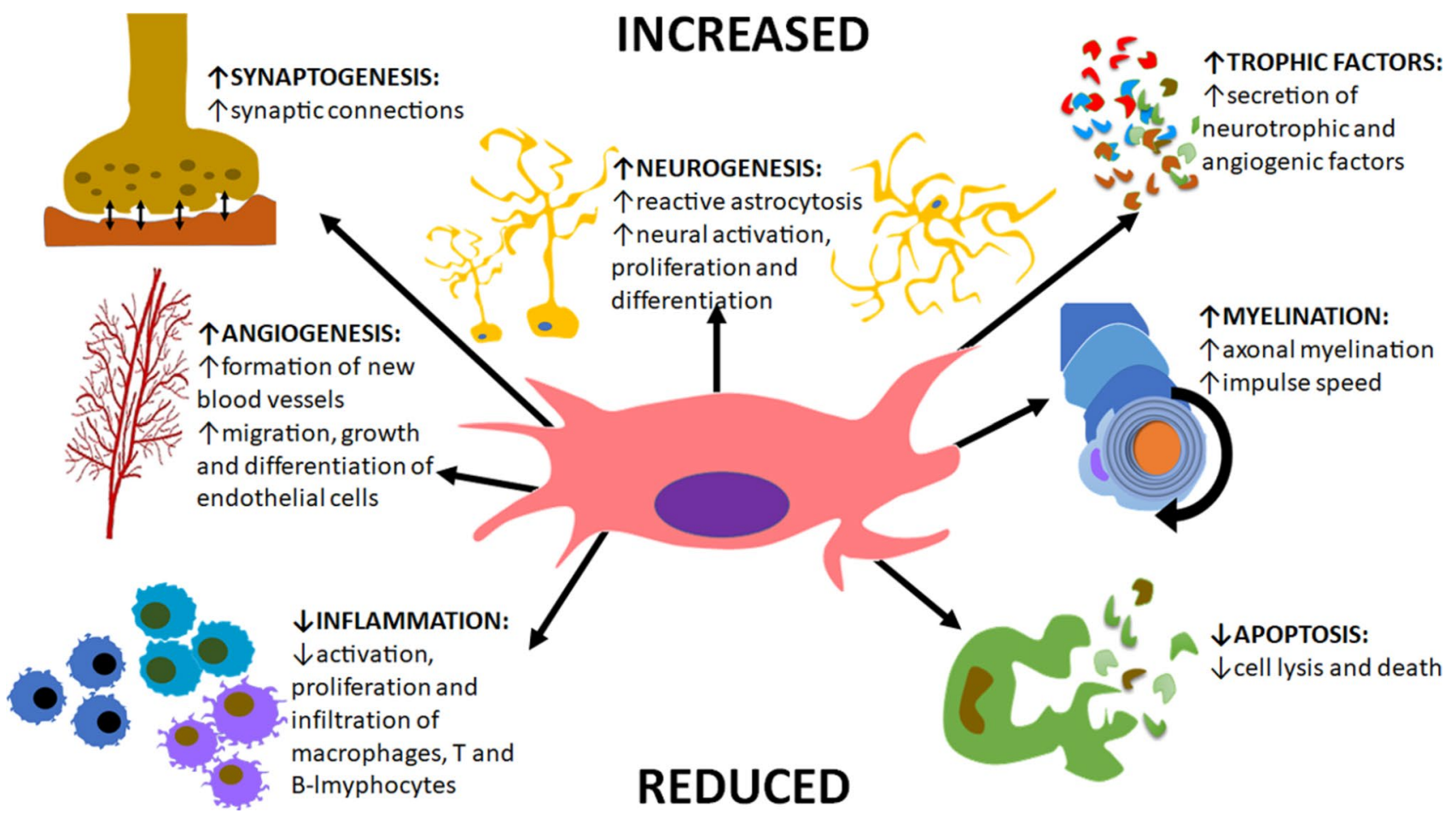

Fig. 1 The function of MSCs play a significant role in the repair and regeneration process and are identified in this diagram. These include the reduction of cell death to continually replace lost cells, the secre-

\section{Methods}

A systematic literature search was performed using electronic databases PMC (PubMed), Cochrane Library, Web of Science and Scopus, to identify recent English language clinical data published between 2014 and 2019. Keyword search strings of (( ("bone marrow-derived mesenchymal stem cell" OR "bone marrow mesenchymal stromal cell" OR "bone marrow stromal cell")) AND ("osteoarthritis" OR "knee osteoarthritis")) AND ("human" OR "clinical"))) AND "intra-articular injection" were applied. Cell dosages were analysed and compared. Despite results being predominantly positive, to increase confidence in clinical efficacy and comparability there needs to be a standardisation of methodologies including follow-up durations and appropriate controls and the application of quantitative outcome measures.

\section{Results}

Using the search terms described returned 139 records; 117 from PMS, 6 from Cochrane Library, 7 from Web of Science and 14 from Scopus respectively. A further five records were identified via other searches. The removal of duplicate records resulted in 133 records being screened for relevance tion of trophic factors which stabilise the extracellular matrix and the suppression of immune cell activation to prevent inflammation. Adapted from: BioExplorer.net [63]

(Fig. 2). The titles and abstracts were screened, and 100 records were removed since they were unrelated, in vitro studies, non-human studies and/or review articles. Of the 23 full-text articles assessed for eligibility, 9 records were excluded due to the methods employing combined treatments, i.e. BM-MSCs administered with chondrocytes or hyaluronic acid, the cells not being bone-marrow derived or the studies being non-knee specific. 14 studies were included in a qualitative synthesis. Due to the low number of published studies, coupled with the diversity of protocols implemented and significant variation in outcome measures applied it was extremely difficult to directly quantitatively compare studies. Thus, a narrative review was felt to be most appropriate to review and present the relevant literature.

\section{Discussion}

\section{BM-MSCs for stimulating regeneration in knee osteoarthritis}

Bone marrow tissue supports the complex microenvironment for numerous cell types and bone marrow aspirate can be used whole, concentrated, or as a source for stem cells [19]. BM-MSCs can be isolated from aspirate and have gained significant attention in the regenerative medicine field [22] 
Fig. 2 PRISMA flowchart of study selection criteria

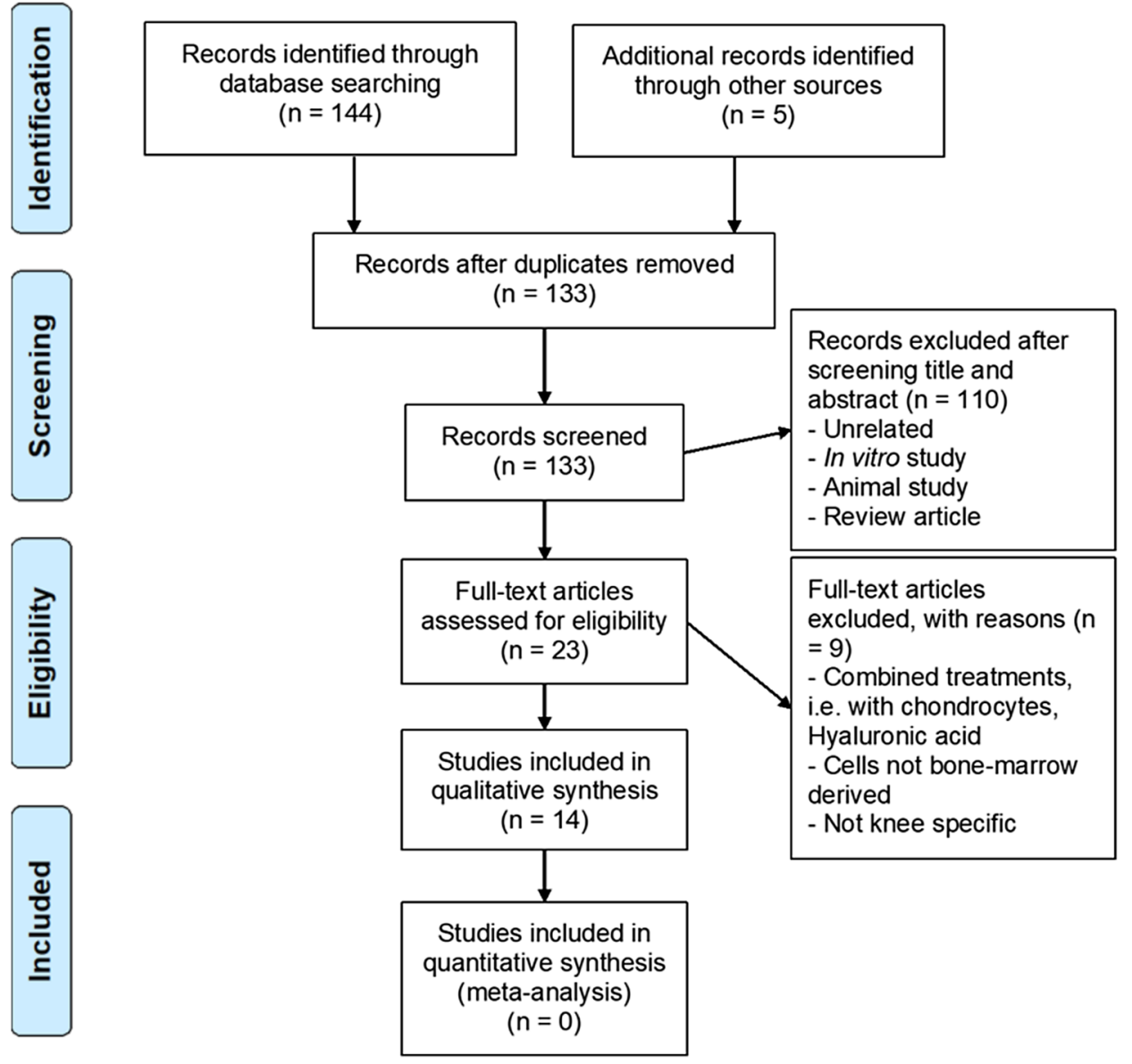

due to their multilineage differentiation potential, immunomodulatory and self-renewal capacities [21, 23].

BM-MSCs promote repair via paracrine signalling mechanisms and the secretion of soluble trophic factors including bone morphogenetic protein-2 (BMP2) and insulin-like growth factor-1 (IGF1) [24]. These factors enhance cellular regeneration and induce bone formation by stimulating proliferation and differentiation of endogenous semi-like progenitors found in most tissues and by decreasing OA inflammatory and immune reactions [25]. BM-MSCs also inhibit T- and B-lymphocyte activation by inhibiting inflammatory cytokine production, thereby preventing immune responses and consequently promoting immune tolerance. Furthermore, BM-MSCs stimulate anti-inflammatory interleukin-1 (IL-1) supporting the generation of anti-inflammatory
T-cells [26]. To guarantee these characteristics and standStem Cell Committee of the International Society for Cellular Therapy (ISCT) has defined MSC criteria (Table 2) to improve the validity and consistency of research trials.

Pre-clinical studies investigating BM-MSCs for cartilage repair in animal models have demonstrated encouraging results (Table 3) [27-33]; subsequently, clinical applications are increasing (Table 4) [30, 34-44]. BMMSCs administered for KOA in clinical patients adhere to damaged tissue surfaces, and differentiate into chondrocytes, resulting in anatomic restoration with significant improvements regarding pain and function [40, 43]. However, some studies have challenged whether BM-MSCs treatments are applicable to all OA grades [45-47]. Across ardise MSC classification, the Mesenchymal and Tissue
Table 2 Committee of the ISCT criteria for the classification of mesenchymal stem cells [66]

\begin{tabular}{l}
\hline Classification of MSCs \\
\hline Fibroblastic-like (spindle-shaped) morphology \\
Plastic-adherent property under standard culture conditions \\
Differentiation potential into osteoblasts, adipocytes, and chondroblasts in vitro \\
Expression of surface markers including CD105; CD73 and; CD90 \\
Lack of expression of: CD45; CD34; CD14; or CD11b; CD79 $\alpha$ or CD19 and; HLA-DR
\end{tabular}




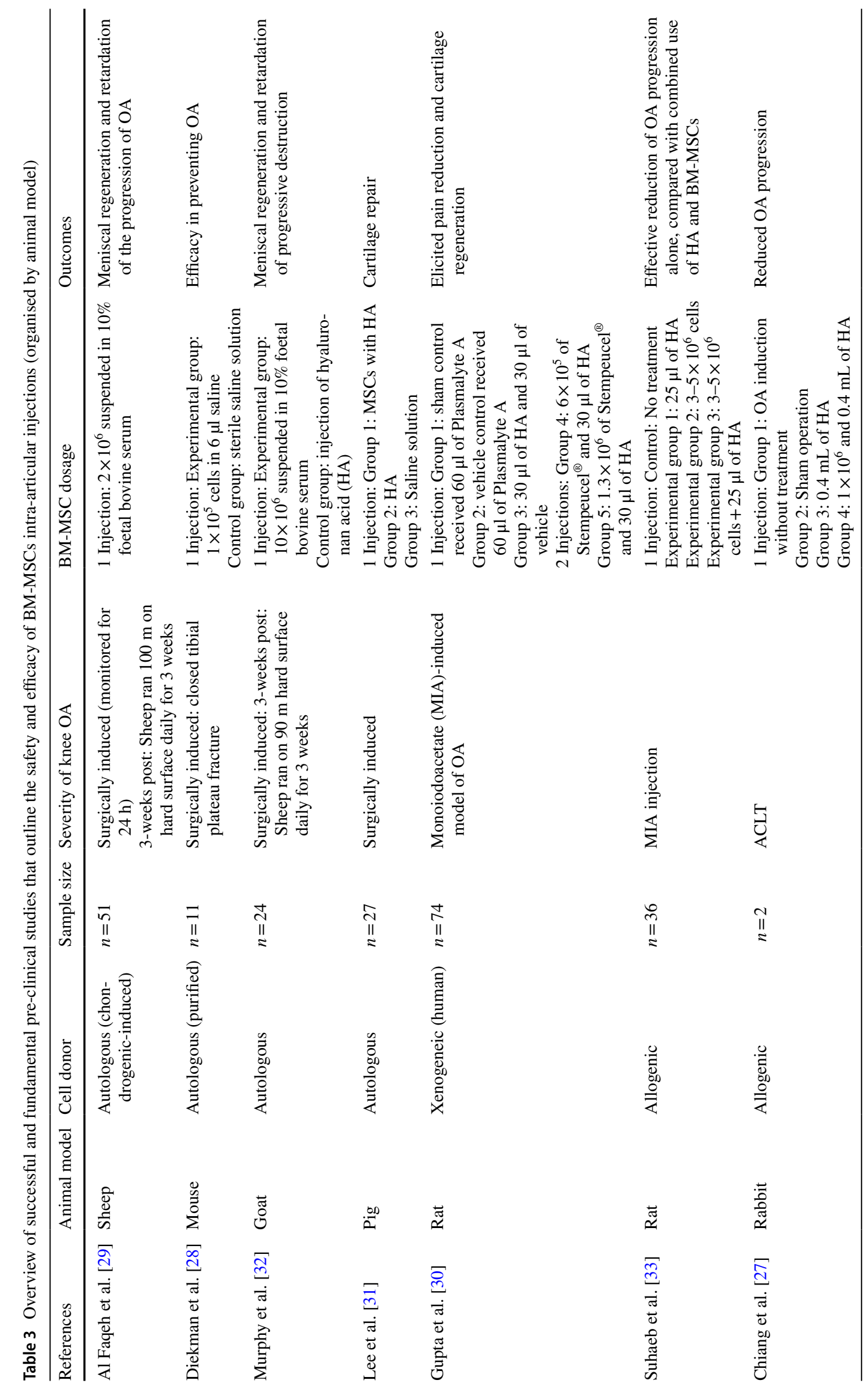




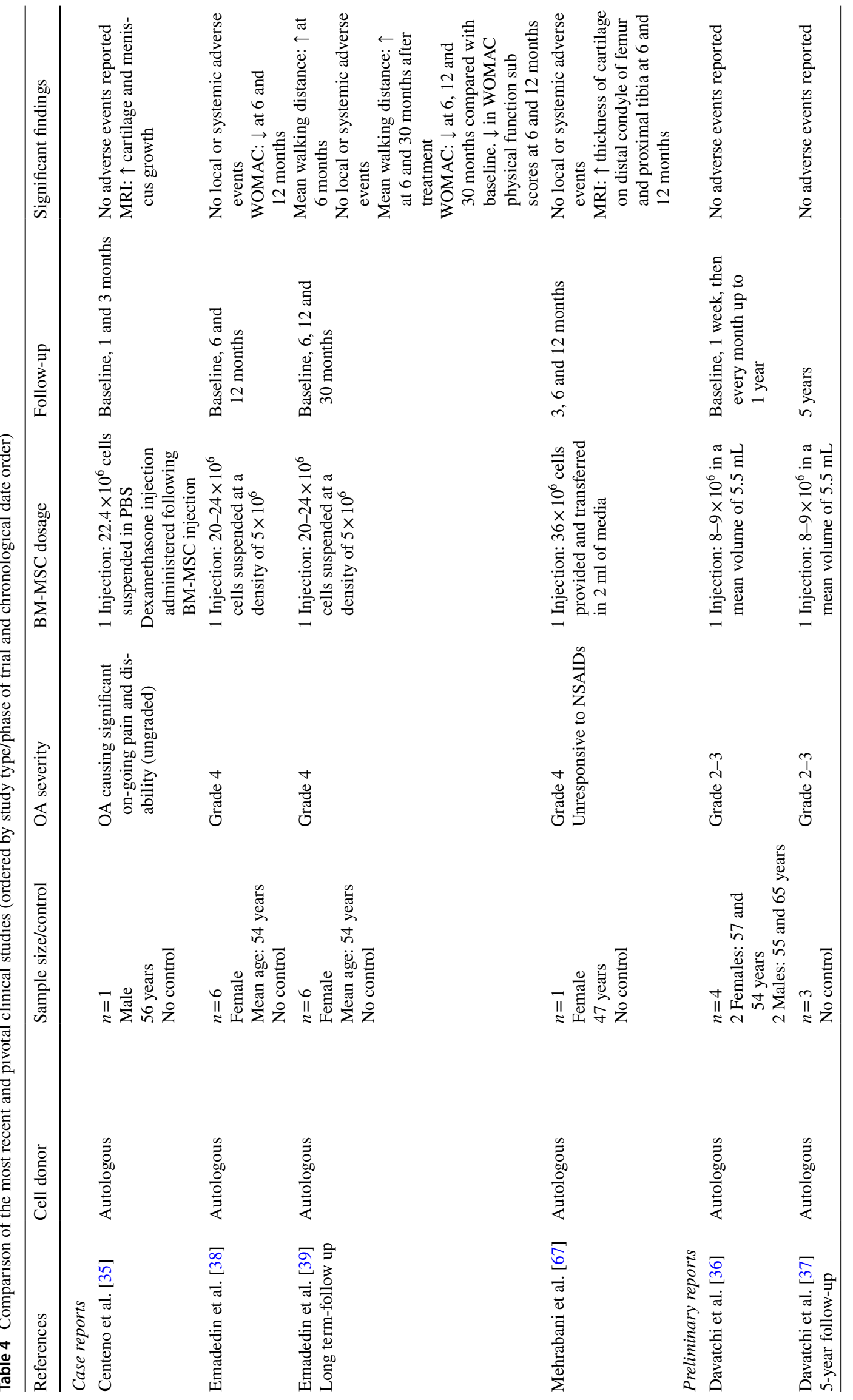




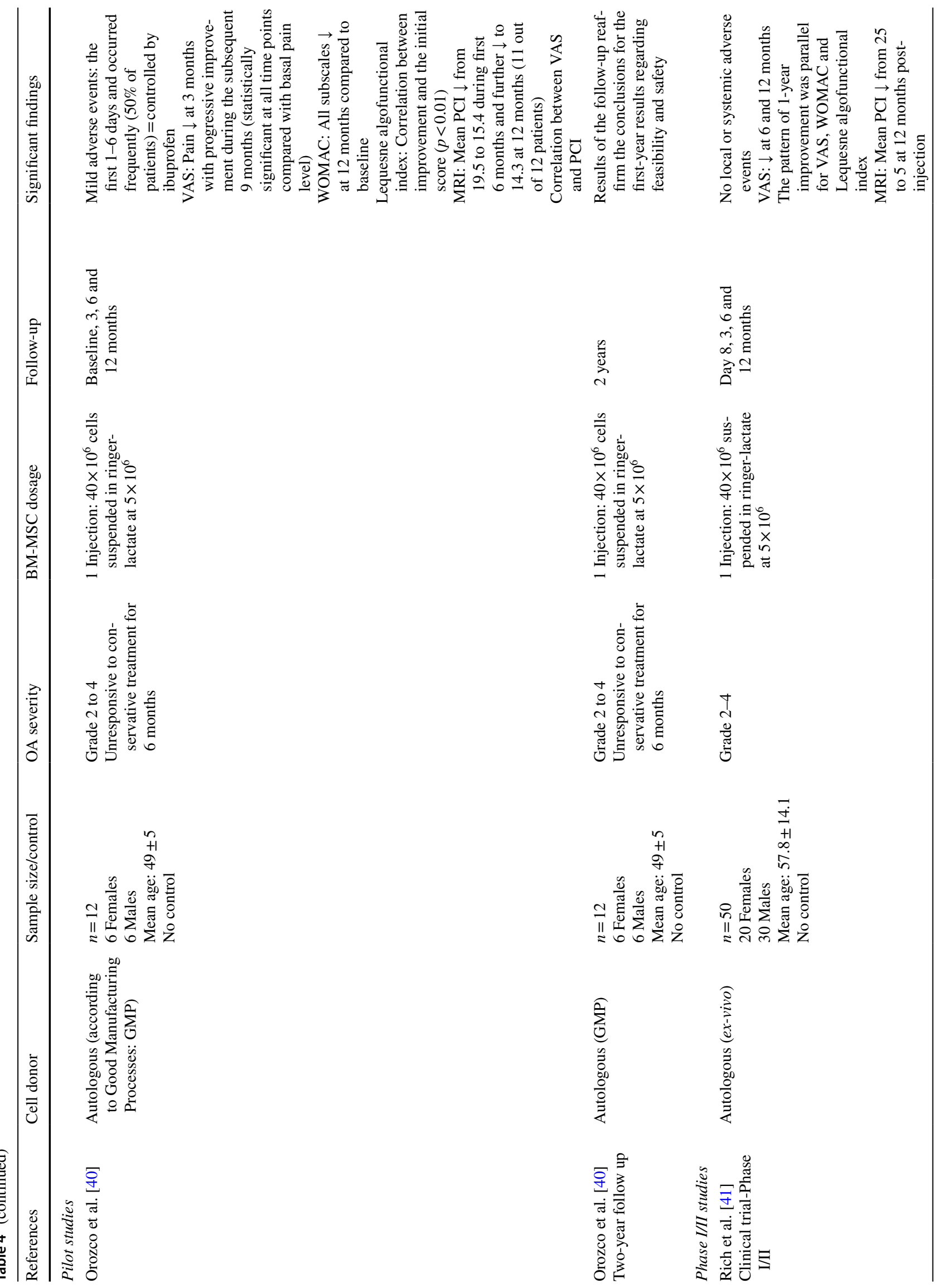




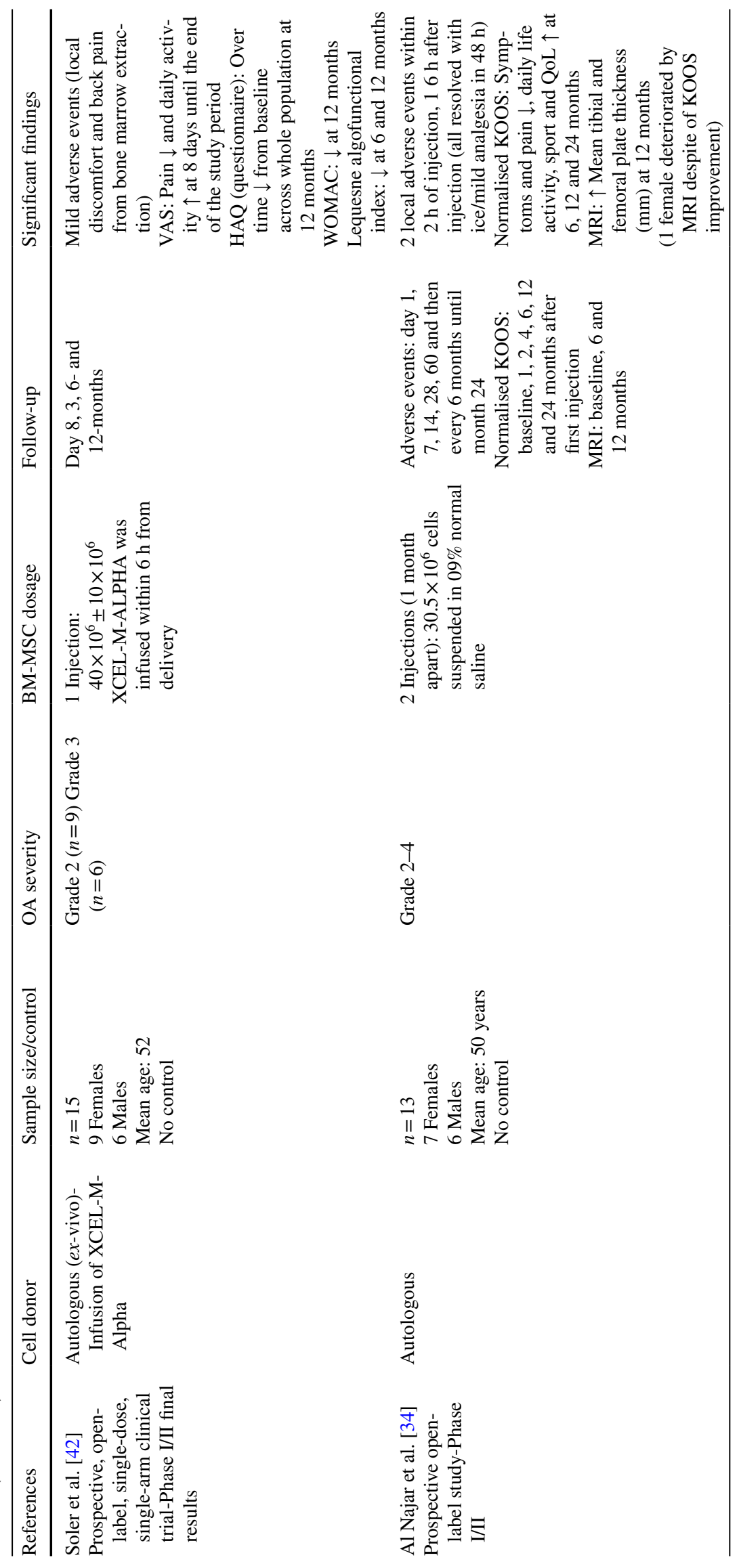




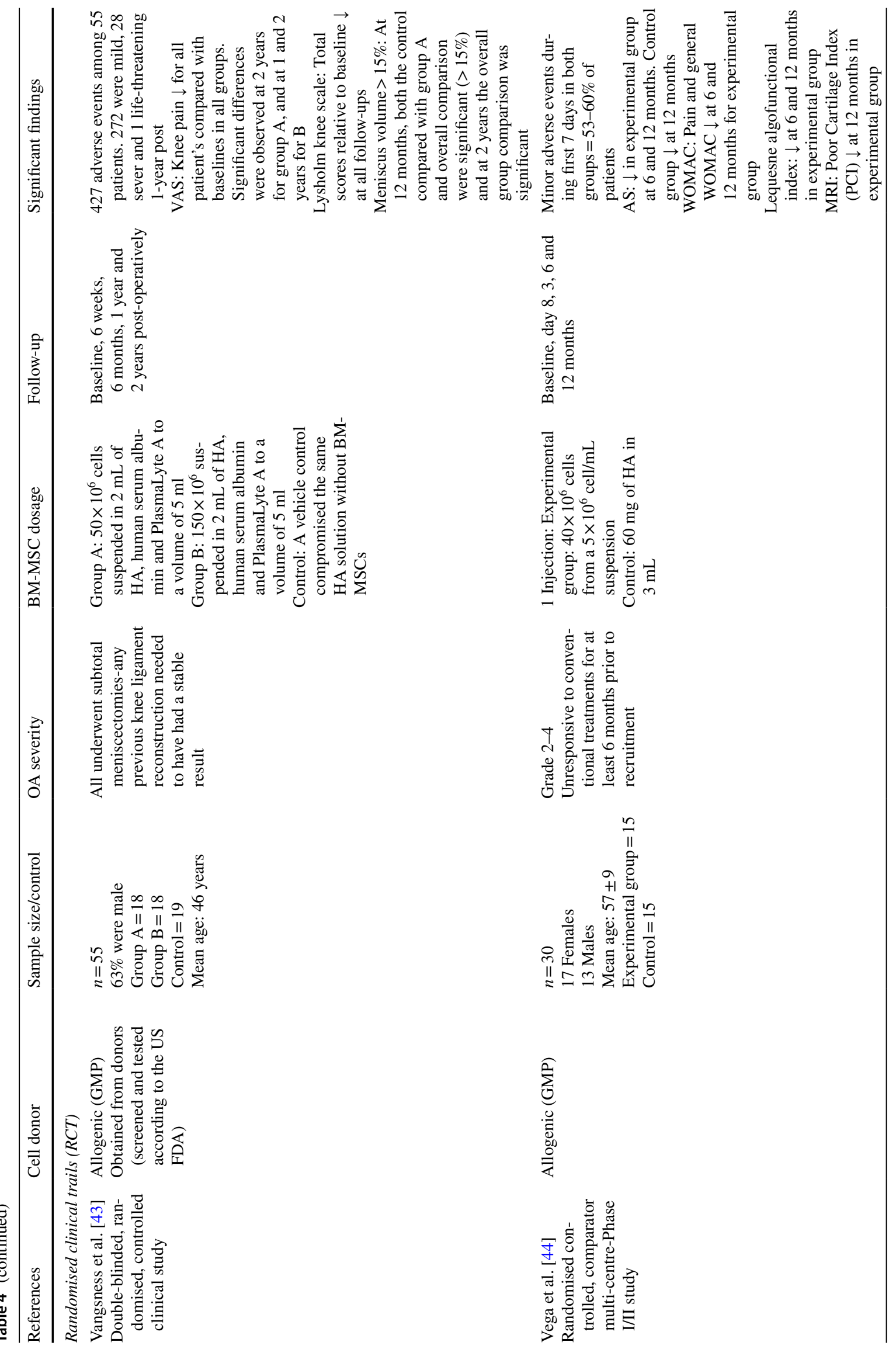




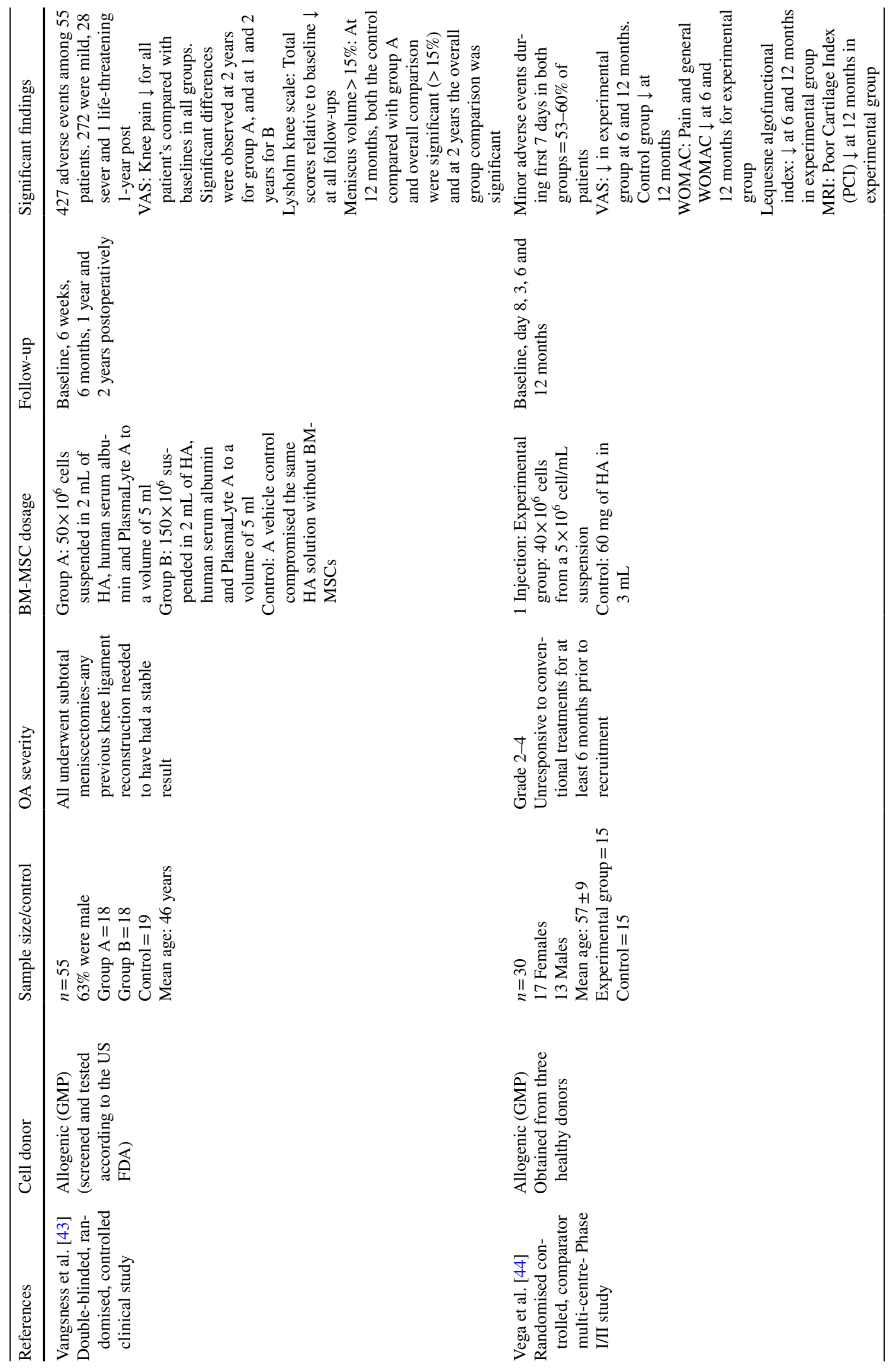




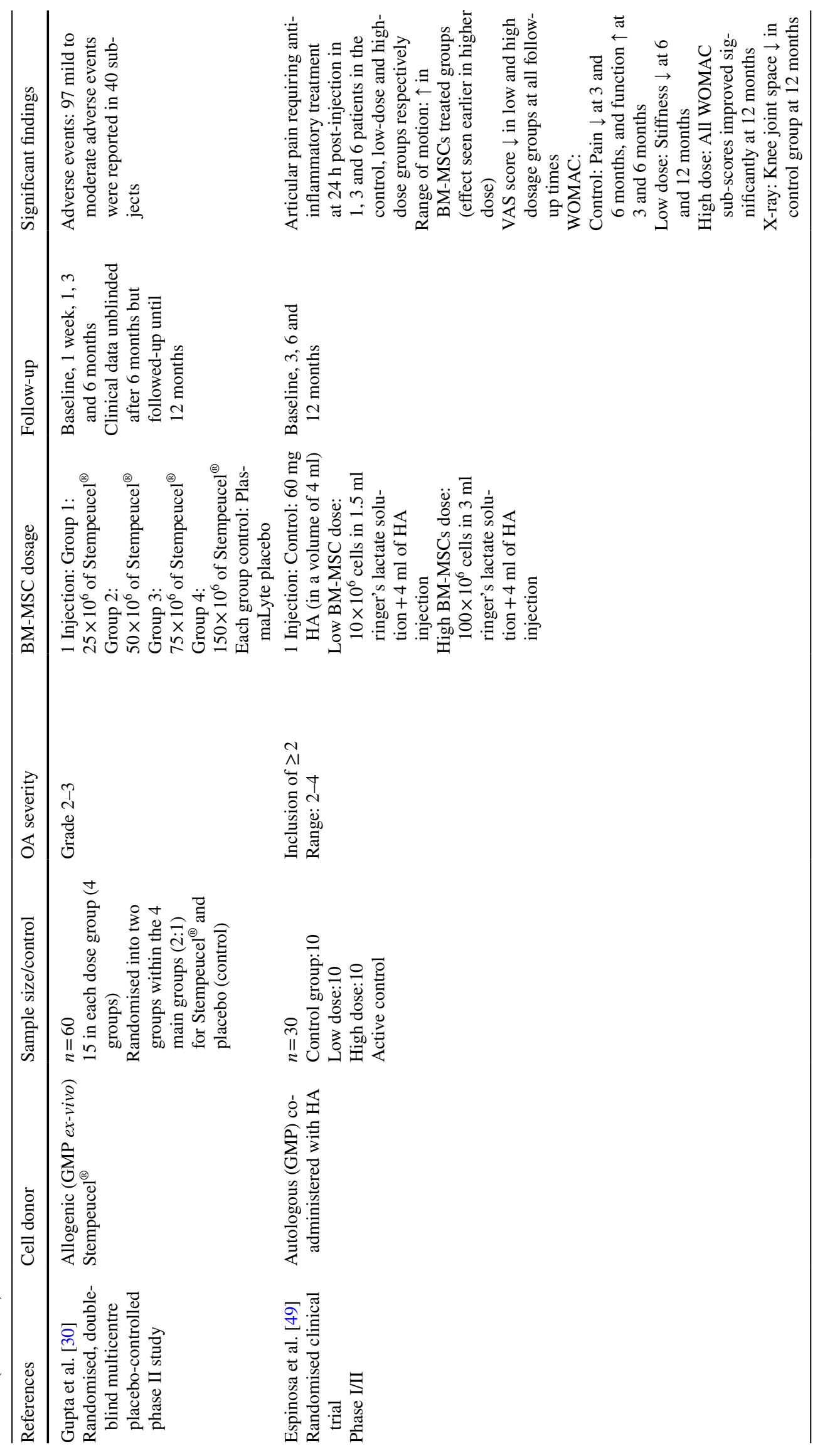


research studies, a variety of outcome measures have been utilised, with some studies reliant upon qualitative questionnaires including The Western Ontario and McMaster Universities Osteoarthritis Index (WOMAC) and Lequesne algofunctional indexes [20] to evaluate success, which may introduce unintended bias [38, 42], due to physicians influencing patient responses. This may be improved by digital administration of questionnaires therefore, it will be completed individually with no external input [48].

Within the literature reviewed, the follow-up periods and outcome criteria varied. Periods up to 12 -month post-injection have been followed, with clinical outcomes including increased cartilage thickness, function and pain measured [34]. Despite reported improvements, not all are significant. Contrastingly, greater BM-MSC longevity with significant changes in both qualitative and quantitative after-effects have been reported [34, 44, 49]. An injection of $40 \times 10^{6}$ cells in 12 patients with advanced KOA displayed significant improvements in VAS and quality of articular cartilage without diminution between a 12 [40] and 24-month follow-up [44]. Davatchi et al. [37] reported a 5-year follow-up post injection of $8-9 \times 10^{6} \mathrm{BM}-\mathrm{MSCs}$ in 4 patients and observed progressive deterioration, although outcomes were improved compared to baseline measurements, suggesting a protective role of BM-MSCs compared to untreated controls. Despite prolonged followup periods, limited patient numbers and lack of in-depth statistical analysis make it difficult to draw robust conclusions regarding the overall therapeutic efficacy [50].

Compared to autologous BM-MSCs, allogenic BMMSCs represent an alternative cell source. Multiple randomised control trials (RCTs) have reported improved outcomes with various doses $\left(25 \times 10^{6}-50 \times 10^{6}\right.$ cells $)$, which are safe and well tolerated, whereas higher doses can produce adverse events [30]. Despite positive trends in similar studies $[43,44]$, few clinical parameters were significantly improved; with no critical changes in X-ray and Magnetic Resonance Imaging (MRI) compared to baseline measurements. Although BM-MSCs are considered poorly immunogenic, allogenic cells may stimulate immune responses and thus, the identification of an optimum dose is crucial for viable treatment strategies [50].

There is currently limited evidence for simultaneous improved clinical outcomes, including pain, function, and cartilage repair. However, improvements following the application of intra-articular BM-MSCs at short-term follow-up have been reported [22]. Several studies have reported improved cartilage thickness; however, meaningful changes in clinical outcomes are sporadic. Moreover, studies using both autologous and allogenic BM-MSCs have been explored within the literature, including coadministered and ex-vivo expanded treatments [30,49].

\section{Critique of BM-MSC intra-articular injections for the treatment of knee osteoarthritis}

Autologous BM-MSC injections are the dominant cell choice in clinical studies reported for treating KOA [51]. In an early case report [35], a single patient was injected with $22.4 \times 10^{6}$ cells suspended in phosphate-buffered saline (PBS) with a dexamethasone post-injection as a differentiating agent [52]. The 3-month follow-up reported no adverse events, with significantly increased cartilage and meniscus growth, with minor improvements in range of motion (ROM) and pain scores. This was the first report of increased meniscus size in humans; however, the methodology lacked specificity and a detailed exploration of the dexamethasone effects was not conveyed [52]. MRI revealed significant cartilage thickening covering the distal femur and proximal tibia at 6 and 12 months, yet symptomatic and functional improvements were not apparent. This study failed to acknowledge potential author or methodological bias and so further pilot and clinical studies are required to replicate meaningful findings.

Davatchi et al. [36] emphasised the safety of BM-MSC injections, claiming marked improvements in (qualitative) outcome parameters, with physical parameters improving to a lesser extent. In comparison to Centeno et al. [35], a lower dosage of $8-9 \times 10^{6}$ BM-MSCs were administered, potentially accounting for lower physical parameter improvements. The follow-up from a 2011 case series [37] argued that the lack of significant outcomes is due to all participants having advanced-stage OA.

Emadedin et al. [38] performed a similar study on a small patient cohort $(n=6)$, using $20-24 \times 10^{6}$ cells, reporting significant improvements in pain and function (WOMAC) at both 6 and 12 months. A long-term follow-up of the same cohort affirmed previous findings, revealing that BM-MSC dosages were safe and therapeutically beneficial. Nevertheless, between 12 and 30 months, therapeutic improvements declined in all individuals, suggesting the need for subsequent administration for prolonged benefit [39].

Despite reported therapeutic benefits of BM-MSCs, the generalisability of the results and techniques used for larger populations with symptomatic KOA is limited. This highlights the requirement for larger, blinded RCTs to improve study comparability and clinical validity. Likewise, within study designs, sample size calculations should be utilised for methodological and ethical reasons. Otherwise, reported findings should be interpreted with caution, as smaller samples may undermine internal and external study validity.

A clinical RCT (phase I/II) of 30 patients with grade $\geq 2$ OA used a sample size calculation that provided an effect size of 0.6 and, a power of $80 \%$ [49]. Group randomisation was performed, potentially facilitating the increased cohort size compared to previous studies 
$(n=30)$, whilst reducing bias [53]. Despite randomisation, the stage of OA was more severe in those receiving low-dose BM-MSCs $\left(10 \times 10^{6}\right)$, which may have prevented these patients achieving more positive outcomes [49]. The study included blinded radiologists to reduce bias (extra KOA MRIs were randomly added during analysis). Ethical issues prevented double-blinding; thus, qualitative clinical scores were compared with objective measures to minimise bias. A control, low-dose and highdose group were followed for 12 months. Outcomes were significant at 3, 6 and 12 months and correlations revealed a significant reduction in low-and-high-dose VAS scores at all time points, which correlated with improved ROM compared to the control group. Importantly, this study co-administered hyaluronic acid (HA) and indicated that a single injection is a safe and feasible procedure, resulting in both clinical and functional improvements; particularly when $100 \times 10^{6}$ cells were administered.

Additional studies using allogenic BM-MSCs also used HA either as a control group or as a cell suspension [43, 44]. However, cells from young, healthy donors may not reflect growth and differentiation characteristics of MSCs from elderly and/or OA patients [54]. Yet, they equally showed improvements in pain and function. Espinosa et al. [55] described a decrease in knee joint space in control groups (HA) at 12 months. Whereas Vangsness et al. and Vega et al. [43, 44] provided MRI analysis using T2 mapping of cartilage and, computational analysis of meniscus volume to assess the effects of BM-MSCs more closely. Vega et al. [44] found significant decreases in poor cartilage index (PCI) at 12-month following injection of $40 \times 10^{6}$ cells. Conversely, a dosage of $50 \times 10^{6}$ cells [43] identified significantly increased meniscus volume (>15\%) at 12 months, which continued 2 years post-injection. Orozco et al. [40] also reported significant improvements in PCI following treatment with autologous BM-MSCs with continued improvement over the 2-year follow-up. A significant correlation between VAS and PCI was reported, demonstrating that both cartilage and pain/function improvements occur simultaneously. This study supersedes previous case reports where results were described as "satisfactory" [39], as cell dosages were larger, the follow-up was longer and the MRI investigation provided robust quantitative analysis.

Alternative methodologies for the application of MSCs into KOA patients exist, however these are more invasive but do allow more specific targeting of focal cartilage defects. Brittberg et al. and Bornes et al. have both comprehensively discussed the use of MSCs (not just limited to BM-MSCs) in the specific treatment of cartilage defects considering multiple therapy options $[56,57]$.

\section{Safety considerations when using BM-MSCs for the treatment of KOA}

The feasibility and safety of both allogenic and autologous cells have been reiterated throughout literature [40, 41]; yet, studies utilising higher doses regularly report increased adverse events. In a sample of 55 patients, 247 adverse events were reported, with one life-threatening, 1-year post-injection when using allogenic BM-MSCs [43]. Minor adverse events, such as post-implantation pain and inflammation, occur with similar frequencies (50\%) between studies using autologous BM-MSCs [40, 49]. Most adverse events were resolved within 24-48 h following treatment with pain medication. Nevertheless, adverse events were not reported in every study [35, 39, 41].

Close attention to adverse events may be key to clinical translation when optimising BM-MSCs as a KOA therapy [51]. The majority of literature reports use of either autologous or allogenic BM-MSCs, however studies have also included infused BM-MSCS [30, 42]. Soler et al. [42] produced an analysis of a prospective, open-label, singlearm clinical trial for the infusion of XCEL-M-Alpha into autologous BM-MSCs. Significant improvements in VAS, WOMAC and Lequesne algofunctional index were observed following a $40 \times 10^{6}$ injection. However, this is one of the first to infuse BM-MSCs and the omission of control groups makes it difficult to evaluate efficacy. The indistinct method regarding infusion is inconsistent with the detailed explanation of cell isolation and expansion and therefore, the methodology may be lacking suitable description. Furthermore, Gupta et al. [30] injected ex vivo expanded, pooled allogenic BM-MSCs (Stempeucel ${ }^{\circledR}$ ) into 60 patients who were split into four different dosage groups (with a control each) in a randomised, double-blinded multicentre placebo-controlled study (RCT). However, unblinding of the trial occurred after 6-month follow-up even though subjective measurements were continuously analysed; after this point, results should be interpreted with circumspection. The therapeutic effect of BM-MSCs was not explored without HA, but both pre-clinical and clinical studies suggest that BM-MSCs co-administered with HA tends to produce greater regenerative benefit [58]. This study also failed to produce any significant outcomes, which was potentially due to the procedure employed with higher dosages and volumes (75 and 150 million cells) being restricted in the limited joint space; possibly causing cell aggregation. The study may have been more valuable if lower dosages were also examined.

Overall, there is moderate-to-high level evidence of safety to recommend therapeutic administration of BMMSCs for KOA, for both animal and human studies; suggesting therapeutic benefit. Several published results, especially conclusions and speculations drawn from case/ preliminary reports, do not have the weight of findings 
compared to RCTs. Therefore, when interpreting results, due diligence is recommended. Furthermore, the significance of experimental outcomes may be influenced by the prevalent study heterogeneity including: the use of a variety of cell doses and donors; variability in functionality and pain scores; severity of KOA; various cell processing methods and differing follow-up periods [59].

Due to the immune-privileged status of MSCs [60], allogenic BM-MSCs show more promise compared to autologous, since they allow manufacturing of large batches or 'off-the-shelf' products in the future [61]. This would enhance the reliability of production whilst decreasing the costs of cell therapies [62], however, long-term efficacy data are warranted.

Conflicting study results may result from methodological heterogeneity or, the limitation of BM-MSCs remaining localised within the tissue. This may be caused by the rapid cycling of synovial fluid or due to large volumes injected into the knee causing cell apoptosis. Despite reported adverse events, the outcomes across the published studies are influential in demonstrating that the benefits may outweigh the treatment risks. Despite BM-MSCs intra-articular injections potentially having a limited therapeutic effect on cartilage volume [51], the clinical and functional outcomes are favourable in patients with chronic KOA. In terms of evaluating BM-MSC efficacy, it may be more applicable for future studies to only focus on long-term, large-scale RCTs as non-RCTs tend to have greater bias and more confounders, affecting the interpretation and validity of efficacy [22]. Future studies need to determine the type and quality of the repaired cartilage tissues, its durability and the association between objective and subjective outcome improvements [22].

\section{Conclusion}

It is apparent that moderate-high cell numbers $\left(40 \times 10^{6}\right)$ are most likely to achieve optimal responses in individuals with grade $\geq 2 \mathrm{KOA}$. The highest number of cells used $\left(100 \times 10^{6}\right)$ also produced significant improvements in KOA, although this came at a cost, with greater risks of adverse events. Therefore, the number and type of cell donor BM-MSCs, the timing of injection, the stage of the disease and the number of injections requires further investigation to achieve optimal therapeutic benefit.

Subsequently, a unified classification of intra-articular dosage and efficacy needs to be agreed before safe and effective treatment can be implemented as a leading regenerative treatment strategy, across all OA populations.
Author contributions All named authors, ECD, NMW and SLW have made substantial contributions to the conception and design of this manuscript. ECD was predominantly responsible for the acquisition of information, all authors were involved in the analysis and interpretation of data. ECD and NMW have been involved in drafting the manuscript, SLW has contributed to the critical revisions to the content. All authors have given their final approval of the version to be published and agree to be accountable for all aspects of the work in ensuring that questions related to the accuracy or integrity of any part of the work are appropriately investigated and resolved.

\section{Compliance with ethical standards}

Conflict of interest The authors have no other relevant affiliations or financial involvement with any organization or entity with a financial interest in or financial conflict with the subject matter or materials discussed in this manuscript. There is no funding to report related to the production of this manuscript.

Funding The author(s) received no specific funding for this work.

Ethical approval Ethics approval was not required for this systematic review.

Open Access This article is licensed under a Creative Commons Attribution 4.0 International License, which permits use, sharing, adaptation, distribution and reproduction in any medium or format, as long as you give appropriate credit to the original author(s) and the source, provide a link to the Creative Commons licence, and indicate if changes were made. The images or other third party material in this article are included in the article's Creative Commons licence, unless indicated otherwise in a credit line to the material. If material is not included in the article's Creative Commons licence and your intended use is not permitted by statutory regulation or exceeds the permitted use, you will need to obtain permission directly from the copyright holder. To view a copy of this licence, visit http://creativecommons.org/licenses/by/4.0/.

\section{References}

1. Cross M, Smith E, Hoy D, Nolte S, Ackerman I, Fransen M et al (2014) The global burden of hip and knee osteoarthritis: estimates from the global burden of disease 2010 study. Ann Rheum Dis 73(7):1323-1330

2. Vos T, Barber RM, Bell B, Bertozzi-Villa A, Biryukov S, Bolliger I et al (2015) Global, regional, and national incidence, prevalence, and years lived with disability for 301 acute and chronic diseases and injuries in 188 countries, 1990-2013: a systematic analysis for the global burden of disease study 2013. Lancet 386(9995):743-800

3. Antony B, Jones G, Jin X, Ding C (2016) Do early life factors affect the development of knee osteoarthritis in later life: a narrative review. Arthritis Res Ther 18(1):1-40

4. Kiadaliri AA, Lamm CJ, de Verdier MG, Engström G, Turkiewicz A, Lohmander LS et al (2016) Association of knee pain and different definitions of knee osteoarthritis with health-related quality of life: a population-based cohort study in southern Sweden. Health Qual Life Outcomes 14(1):1-7

5. Murphy L, Schwartz TA, Helmick CG, Renner JB, Tudor G, Koch $\mathrm{G}$ et al (2008) Lifetime risk of symptomatic knee osteoarthritis. Arthritis Care Res 59(9):1207-1213 
6. Araujo I, Castro M, Daltro C, Matos M (2016) Quality of life and functional independence in patients with osteoarthritis of the knee. Knee Surg Relat Res 28(3):219-224

7. Blagojevic M, Jinks C, Jeffery A, Jordan KP (2010) Risk factors for onset of osteoarthritis of the knee in older adults: a systematic review and meta-analysis. Osteoarthr Cartil 18(1):24-33. https:// doi.org/10.1016/j.joca.2009.08.010

8. Driban J, McAlindon T, Amin M, Price L, Eaton C, Davis J et al (2018) Risk factors can classify individuals who develop accelerated knee osteoarthritis: data from the osteoarthritis initiative. J Orthop Res 36(3):876-880

9. Silverwood V, Blagojevic-Bucknall M, Jinks C, Jordan JL, Protheroe J, Jordan KP (2015) Current evidence on risk factors for knee osteoarthritis in older adults: a systematic review and metaanalysis. Osteoarthr Cartil 23(4):507-515

10. Ashkavand Z, Malekinejad H, Vishwanath B (2013) The pathophysiology of osteoarthritis. J Pharm Res 7(1):132-138

11. Xing D, Wang B, Zhang W, Yang Z, Hou Y, Chen Y et al (2017) Intra-articular platelet-rich plasma injections for knee osteoarthritis: An overview of systematic reviews and risk of bias considerations. Int J Rheum Dis 20(11):1612-1630

12. Petecchia L, Viti F, Sbrana F, Vassalli M, Gavazzo P (2017) A biophysical approach to quantify skeletal stem cells trans-differentiation as a model for the study of osteoporosis. Biophys Chem 229:84-92

13. Glyn-Jones S, Palmer AJR, Agricola R, Price AJ, Vincent TL, Weinans H et al (2015) Osteoarthritis. Lancet 386(9991):376-387

14. Tummala S, Bay-Jensen A-C, Karsdal MA, Dam EB (2011) Diagnosis of osteoarthritis by cartilage surface smoothness quantified automatically from knee MRI. Cartilage 2(1):50-59

15. Guermazi A, Hayashi D, Roemer F, Felson DT, Wang K, Lynch J et al (2015) Severe radiographic knee osteoarthritis-does Kellgren and Lawrence grade 4 represent end stage disease?-the MOST study. Osteoarthr Cartil 23(9):1499-1505

16. McAlindon TE, LaValley MP, Harvey WF, Price LL, Driban JB, Zhang M et al (2017) Effect of intra-articular triamcinolone vs saline on knee cartilage volume and pain in patients with knee osteoarthritis. JAMA 317(19):1967-1975

17. Rillo O, Riera H, Acosta C, Liendo V, Bolaños J, Monterola L et al (2016) PANLAR consensus recommendations for the management in osteoarthritis of hand, hip, and knee. JCR J Clin Rheumatol 22(7):345-354

18. Wise J (2017) Steroid injections for knee osteoarthritis are not supported by study. BMJ 357:1-1

19. Shariatzadeh M, Song J, Wilson SL (2019) The efficacy of different sources of mesenchymal stem cells for the treatment of knee osteoarthritis. Cell Tissue Res 378(3):339-410

20. O'Connell B, Wragg NM, Wilson SL (2019) The use of PRP injections in the management of knee osteoarthritis. Cell Tissue Res 376(2):143-152

21. Chen F, Tuan R (2008) Mesenchymal stem cells in arthritic diseases. Arthritis Res Ther 10(5):1-12

22. Chul-won D, Yong-beom Park M, Seong H, Kim H, Han-jun Lee D (2019) Intra-articular mesenchymal stem cells in osteoarthritis of the knee: a systematic review of clinical outcomes and evidence of cartilage repair. Arthrosc J Arthrosc Relat Surg 35(1):277-288

23. Pastides P, Chimutengwende-Gordon M, Maffulli N, Khan W (2013) Stem cell therapy for human cartilage defects: a systematic review. Osteoarthr Cartil 21(5):646-654

24. Ullah I, Subbarao R, Rho G (2015) Human mesenchymal stem cells-current trends and future prospective. Biosci Rep 35(2):1-1962

25. Leuning DG, Beijer NRM, Du Fossé NA, Vermeulen S, Lievers E, Van Kooten C et al (2018) The cytokine secretion profile of mesenchymal stromal cells is determined by surface structure of the microenvironment. Sci Rep 8:1-26
26. Li H, Shen S, Fu H, Wang Z, Li X, Sui X et al (2019) Immunomodulatory functions of mesenchymal stem cells in tissue engineering. Stem Cells Int 2019:1-18

27. Chiang E-R, Ma H-L, Wang J-P, Liu C-L, Chen T-H, Hung S-C (2016) Allogeneic mesenchymal stem cells in combination with hyaluronic acid for the treatment of osteoarthritis in rabbits. PLoS ONE 11(e0149835):1-15

28. Diekman B, Wu C-L, Louer C, Furman B, Huebner J, Kraus V et al (2013) Intra-articular delivery of purified mesenchymal stem cells from C57BL/6 or MRL/MpJ superhealer mice prevents posttraumatic arthritis. Cell Transplant 22(8):1395-1408

29. Al Faqeh H, Nor Hamdan BMY, Chen HC, Aminuddin BS, Ruszymah BHI (2012) The potential of intra-articular injection of chondrogenic-induced bone marrow stem cells to retard the progression of osteoarthritis in a sheep model. Exp Gerontol 47(6):458-464

30. Gupta PK, Chullikana A, Rengasamy M, Shetty N, Pandey V, Agarwal V et al (2016) Efficacy and safety of adult human bone marrow-derived, cultured, pooled, allogeneic mesenchymal stromal cells (Stempeucel ${ }^{\circledR}$ ): preclinical and clinical trial in osteoarthritis of the knee joint. Arthritis Res Ther 18(1):1-18

31. Lee KBL, Hui JHP, Song IC, Ardany L, Lee EH (2007) Injectable mesenchymal stem cell therapy for large cartilage defectsa porcine model. Stem Cells 25(11):2964-2971

32. Murphy JM, Fink DJ, Hunziker EB, Barry FP (2003) Stem cell therapy in a caprine model of osteoarthritis. Arthritis Rheumatol 48(12):3464-3474

33. Suhaeb A, Naveen S, Mansor A, Kamarul T (2012) Hyaluronic acid with or without bone marrow derived-mesenchymal stem cells imrpoves osteoarthritic knee changes in rat model: a preliminary report. Indian J Exp Biol 50(6):383-390

34. Al-Najar M, Khalil H, Al-Ajlouni J, Al-Antary E, Hamdan M, Rahmeh R et al (2017) Intra-articular injection of expanded autologous bone marrow mesenchymal cells in moderate and severe knee osteoarthritis is safe: a phase I/II study. J Orthop Surg Res 12(190): 1-6

35. Centeno C, Busse D, Kisiday J, Keohan C, Freeman M (2008) Increased knee cartilage volume in degenerative joint disease using percutaneously implanted, autologous mesenchymal stem cells, platelet lysate and dexamethasone. Am J Case Rep 11(3):343-353

36. Davatchi F, Abdollahi BS, Mohyeddin M, Nikbin B (2011) Mesenchymal stem cell therapy for knee osteoarthritis. Preliminary report of four patients. Int J Rheum Dis 14(12):211-215

37. Davatchi F, Sadeghi Abdollahi B, Mohyeddin M, Nikbin B (2016) Mesenchymal stem cell therapy for knee osteoarthritis: 5 years follow-up of three patients. Int J Rheum Dis 19(3):219-225

38. Emadedin M, Aghdami N, Taghiyar L, Fazeli R, Moghadasali $\mathrm{R}$, Jahangir $\mathrm{S}$ et al (2012) Intra-articular injection of autologous mesenchymal stem cells in six patients with knee osteoarthritis. Arch Iran Med 15(7):422-428

39. Emadedin M, Hhorbani L, Fazeli R, Mohseni F, Moghadasali R, Mardpour S (2015) Long-term follow-up of intra-articular injection of autologous mesenchymal stem cells in patients with knee, ankle, or hip osteoarthritis. Arch Iran Med 18(6):336-344

40. Orozco L, Munar A, Soler R, Alberca M, Soler F, Huguet M et al (2013) Treatment of knee osteoarthritis with autologous mesenchymal stem cells. Transplant J 95(12):1535-1541

41. Rich S, Munar A, Soler R, Peirau X, Huguet M, Alberca M et al (2015) Stem cell research and therapy treatment of knee osteoarthritis with autologous expanded bone marrow mesenchymal stem cells : 50 cases clinical and MRI results at one year follow-up. J Stem Cell Res Ther 95(12):1535-1541

42. Soler R, Orozco L, Munar A, Huguet M, López R, Vives J et al (2016) Final results of a phase I-II trial using ex vivo expanded 
autologous mesenchymal stromal cells for the treatment of osteoarthritis of the knee confirming safety and suggesting cartilage regeneration. Knee 23(4):647-654

43. Vangsness T, Farr J, Boyd J, Dellaero D, Mills R, LeRoux-Williams M (2014) Adult human mesenchymal stem cells delivered via intra-articular injection of the knee following partial medial meniscectomy. J Bone Jt Surg 96(2):90-98

44. Vega A, Martín-Ferrero MA, Del Canto F, Alberca M, García V, Munar A et al (2015) Treatment of knee osteoarthritis with allogeneic bone marrow mesenchymal stem cells. Transplantation 99(8):1681-1690

45. Chahla J, Piuzzi NS, Mitchell JJ, Dean CS, Pascual-Garrido C, LaPrade RF et al (2016) Intra-articular cellular therapy for osteoarthritis and focal cartilage defects of the knee. J Bone Jt Surg 98(18):1511-1521

46. Cui G-H, Wang YY, Li C-J, Shi C-H, Wang W-S (2016) Efficacy of mesenchymal stem cells in treating patients with osteoarthritis of the knee: a meta-analysis. Exp Ther Med 12(5):3390-3400

47. Pas H, Winters M, Haisma H, Koenis M, Tol J, Moen M (2017) Stem cell injections in knee osteoarthritis: a systematic review of the literature. Br J Sports Med 51(15):1125-1133

48. Bolarinwa O (2015) Principles and methods of validity and reliability testing of questionnaires used in social and health science researches. Niger Postgrad Med J 22(4):195-201

49. Lamo-Espinosa JM, Mora G, Blanco JF, Granero-Moltó F, NúñezCórdoba JM, López-Elío S et al (2016) Intra-articular injection of two different doses of autologous bone marrow mesenchymal stem cells versus hyaluronic acid in the treatment of knee osteoarthritis: long-term follow up of a multicenter randomized controlled clinical trial (phase I/II). J Transl Med BioMed Cent 16(1):1-9

50. Lopa S, Colombini A, Moretti M, de Girolamo L (2019) Injective mesenchymal stem cell-based treatments for knee osteoarthritis: from mechanisms of action to current clinical evidences. Knee Surg Sport Tr A 27(6):2003-2020

51. Iijima H, Isho T, Kuroki H, Takahashi M, Aoyama T (2018) Effectiveness of mesenchymal stem cells for treating patients with knee osteoarthritis: a meta-analysis toward the establishment of effective regenerative rehabilitation. NPJ Regen Med 3:1-15

52. Bosnakovski D, Mizuno M, Kim G, Takagi S, Okumura M, Fujinaga T (2006) Chondrogenic differentiation of bovine bone marrow mesenchymal stem cells (MSCs) in different hydrogels: Influence of collagen type II extracellular matrix on MSC chondrogenesis. Biotechnol Bioeng 93(6):1152-1163

53. Gluud L (2006) Bias in clinical intervention research. Am J Epidemiol 163(6):493-501

54. Ganguly P, El-Jawhari JJ, Giannoudis PV, Burska AN, Ponchel F, Jones EA (2017) Age-related changes in bone marrow mesenchymal stromal cells. Cell Transplant 26(9):1520-1529

55. Lamo-Espinosa JM, Mora G, Blanco JF, Granero-Moltó F, NúñezCórdoba JM, López-Elío S et al (2018) Intra-articular injection of two different doses of autologous bone marrow mesenchymal stem cells versus hyaluronic acid in the treatment of knee osteoarthritis: long-term follow up of a multicenter randomized controlled clinical trial (phase I/II). J Transl Med 16(1):1-9

56. Bornes TD, Adesida AB, Jomha NM (2014) Mesenchymal stem cells in the treatment of traumatic articular cartilage defects: a comprehensive review. Arthritis Res Ther 16(5):1-62

57. Brittberg M, Gomoll AH, Canseco JA, Far J, Lind M, Hui J (2016) Cartilage repair in the degenerative ageing knee. Acta Orthop 87(sup 363):26-38

58. Sato M, Uchida K, Nakajima H, Miyazaki T, Guerrero A, Watanabe $S$ et al (2012) Direct transplantation of mesenchymal stem cells into the knee joints of hartley strain guinea pigs with spontaneous osteoarthritis. Arthritis Res Ther 14(1):1-9

59. Shin Y, Yoon J, Kim H (2018) Intra-articular injection of bone marrow-derived mesenchymal stem cells leading to better clinical outcomes without difference in MRI outcomes from baseline in patients with knee osteoarthritis. Knee Surg Relat Res 30(3):206-214

60. Glenn JD (2014) Mesenchymal stem cells: emerging mechanisms of immunomodulation and therapy. World J Stem Cells 6(5):526-539

61. Shah K, Zhao A, Sumer H (2018) New approaches to treat osteoarthritis with mesenchymal stem cells. Stem Cells Int 2018:1-9

62. De Bari C, Roelofs A (2018) Stem cell-based therapeutic strategies for cartilage defects and osteoarthritis. Curr Opin Pharmacol 40:74-80

63. BioExplorer.net (2016) Mesenchymal stem cells (MSCs): overview, properties, functions and therapies. https://www.bioexplore r.net/mesenchymal-stem-cells.html/

64. Adatia A, Rainsford K, Kean W (2012) Osteoarthritis of the knee and hip. Part I: aetiology and pathogenesis as a basis for pharmacotherapy. J Pharm Pharmacol 64(5):617-625

65. Palmer K (2012) Occupational activities and osteoarthritis of the knee. Br Med Bull 102:147-170

66. Dominici M, Le Blanc K, Mueller I, Slaper-Cortenbach I, Marini F, Krause D et al (2006) Minimal criteria for defining multipotent mesenchymal stromal cells. The International Society for Cellular Therapy position statement. Cytotherapy 8(4):315-317

67. Mehrabani D, Jaberi FM, Zakerinia M (2016) The healing effect of bone marrow-derived stem cells in knee osteoarthritis: a case report. World J Plast Surg 5(2):168-174

Publisher's Note Springer Nature remains neutral with regard to jurisdictional claims in published maps and institutional affiliations. 